\title{
Role of p11 in Cellular and Behavioral Effects of 5-HT4 Receptor Stimulation
}

\author{
Jennifer L. Warner-Schmidt, ${ }^{1}$ Marc Flajolet, ${ }^{1}$ Abigail Maller, ${ }^{1}$ Emily Y. Chen, ${ }^{1}$ Hongshi Qi, ${ }^{2}$ Per Svenningsson,,${ }^{1,2}$ and \\ Paul Greengard ${ }^{1}$ \\ ${ }^{1}$ Laboratory of Molecular and Cellular Neuroscience, The Rockefeller University, New York, New York 10065, and ${ }^{2}$ Center for Molecular Medicine, \\ Department of Physiology and Pharmacology, Karolinska Institute, SE-171 77 Stockholm, Sweden
}

\begin{abstract}
p11 (S100A10), a member of a large family of S100 proteins, interacts with serotonin receptor 1B (5-HTR1B), modulates 5-HT1B receptor signal transduction, and is required for antidepressant responses to activation of this receptor. In the current study, we investigated the specificity of the interaction between 5-HTR1B and p11 by screening brain-expressed S100 proteins against serotonin and noradrenergic receptors. The data indicate that $\mathrm{p} 11$ is unique among its family members for its interactions with defined serotonin receptors. We identify a novel p11-interacting receptor (5-HTR4) and characterize the interaction between p11 and 5-HTR4, demonstrating that (1) p11 and 5-HTR4 mRNA and protein are coexpressed in brain regions that are relevant for major depression, (2) p11 increases 5-HTR4 surface expression and facilitates 5-HTR4 signaling, and (3) p11 is required for the behavioral antidepressant responses to 5-HTR4 stimulation in vivo. The essential role played by 11 in modulating signaling through 5-HT4 as well as 5-HT1B receptors supports the concept that this protein may be a key determinant of vulnerability to depression.
\end{abstract}

Key words: antidepressant; depression; RS67333; S100A10; serotonin receptor; agonist

\section{Introduction}

Depression is a widespread and debilitating disease whose underlying pathophysiology remains unknown. Most of the current treatments for depression and anxiety increase serotonergic neurotransmission (Barnes and Sharp, 1999). p11 was recently identified as a new antidepressant target based on its interactions with the serotonin system via serotonin-1B receptors (5-HTR1B) (Svenningsson et al., 2006). p11 is downregulated in depressivelike states in rodents and humans, and is upregulated following antidepressant treatment. In behavioral assays, p11 knock-out (KO) mice display a depressive-like phenotype and are less sensitive to antidepressants and the 5-HTR1B agonist anpirtoline. Transgenic mice overexpressing p11 behave as if they have already received an antidepressant.

There are several classes of serotonin receptors, and the ones that mediate antidepressant effects are not clearly delineated. There are seven 5-HTR subtypes that have been classified based on the second messenger transduction systems used. All but 5-HTR3 are G-protein-coupled receptors. Noradrenergic receptors are also implicated in the actions of antidepressant treatments. Noradrenaline reuptake inhibitors (NRIs) are also effective therapies for some depressed patients.

\section{Received Nov. 6, 2008; revised Jan. 6, 2009; accepted Jan. 12, 2009}

This work was supported by grants from The Skirball Foundation and The Zilkha Foundation and by United States Army Medical Research Acquisition Activity Grant W81XWH-08-1-0111. We thank Drs. Eric F. Schmidt and Nathaniel Heintz for providing the 5-HTR4 BAC transgenic mouse line used in our studies and John Marshall for technical assistance.

Correspondence should be addressed to Dr. Paul Greengard, 1230 York Avenue, Box No. 296, New York, NY 10065. E-mail: greengard@rockefeller.edu.

DOI:10.1523/JNEUROSCI.5343-08.2009

Copyright $\odot 2009$ Society for Neuroscience $\quad$ 0270-6474/09/291937-10\$15.00/0 p11 (also called S100A10) is one of 21 members of a large family of S100 calcium effector proteins that perform a variety of functions in the CNS, including regulation of kinase activity, phosphorylation of target proteins, and endosomal function (Donato, 1999; Heizmann et al., 2002). p11 is involved in trafficking receptors (e.g., 5-HTR1B) and channels to the cell surface (Svenningsson and Greengard, 2007). Currently, S100 $\beta$ is the only other S100 family member that has been linked to depression. Increased serum levels of $S 100 \beta$ have been proposed as a biomarker for melancholic depression (Grabe et al., 2001; Rothermundt et al., 2001), but evidence for a possible involvement of $\mathrm{S} 100 \beta$ in the pathophysiology of the disease is lacking.

A major limitation of the currently available antidepressant therapies is that they require weeks or months to produce therapeutic effects. Activation of 5-HTR4, in contrast, produces a rapid antidepressant response in rodent models of depression (Lucas et al., 2007). However, 5-HTR4 is expressed in tissues outside the CNS, such as in the gastrointestinal system (Tonini and Pace, 2006), complicating analysis of its utility as an effective antidepressant target. Proteins that interact with and regulate 5-HTR4 function represent potential novel antidepressant targets.

In the present study, we aimed to determine the specificity of the interaction between 5-HTR1B and p11 by using a matrix approach to screen for interactions between brain-expressed S100 proteins and serotonin receptors. We identified the interaction between 5-HTR4 and p11 as being the strongest one, and investigated colocalization of p11 and 5-HTR4 mRNA and protein in regions of the mouse brain that have been linked to depression. We evaluated the ability of p11 to regulate surface expression of 5-HTR4 in vitro and in vivo. Finally, in rodent models 
of depression and anxiety, we asked whether the antidepressant actions of 5-HTR4 stimulation require p11. The findings from this study indicate that $\mathrm{p} 11$ exerts its antidepressant activity, in part, via interactions with 5-HTR4 and underscore the importance of p11 as a potential antidepressant target.

\section{Materials and Methods}

Analysis of 5-HT receptor/S100 interactions. We used the yeast two-hybrid GAL4-based system to screen the 5-HT receptor family for possible interactions within the S100 family. The amino acids corresponding to the entire third intracellular loop of each of the 5-HT receptors and noradrenergic receptors were subcloned into a pAS2 derived plasmid and used as baits. The full-length sequences corresponding to the $S 100$ proteins were subcloned into a pACT2 plasmid and used as preys. The bait and the prey plasmids were transformed into CG1945 and Y187 yeast strains respectively and streaked onto selective medium. The matrix analysis was performed by streaking each of the bait transformants as patches onto rich medium plates (YPD) and by mixing them with each of the prey transformants. Cells were allowed to mate for $16 \mathrm{~h}$, then replica-plated for the selection of diploid cells. After $2 \mathrm{~d}$ at $30^{\circ} \mathrm{C}$, lifts of plates were moved onto nylon membranes for LacZ reporter analysis. Empty pAS2 and PACT2 plasmids were used as negative controls. Positive interactions were reconfirmed using two different bait and prey yeast clones.

Recombinant protein preparation and GST pull-down. The specific interaction between glutathione $S$-transferase (GST)-5-HTR4 third intracellular loop and S100A10 was biochemically investigated with GST pulldown assays. After transformation in Escherichia coli BL21 (DE3) pLysS, expression of protein was induced at midlog phase, by adding $1 \mathrm{~mm}$ isopropyl $\{\beta\}$-D-thiogalactoside. GST-fusion proteins were purified by affinity chromatography by using glutathione-Sepharose beads. Fulllength S100A10 was subcloned into a TA-cloning plasmid. In vitro transcription-translation of S100A10 was performed in the presence of $\left[\mathrm{S}^{35}\right]$-methionine. Loaded GST-Sepharose beads were incubated with in vitro radio-labeled S100A10, and the bound proteins were analyzed by SDS-PAGE.

Biotinylation. COS7 cells were cotransfected with HA-tagged 5-HTR4 and either p1 1 or empty vector control. $48 \mathrm{~h}$ after transfection, cells were washed in ice-cold PBS/CM (PBS with $1 \mathrm{~mm} \mathrm{MgCl}_{2}$ and $1.3 \mathrm{~mm} \mathrm{CaCl}_{2}$ ), incubated for $30 \mathrm{~min}$ in $0.5 \mathrm{mg} / \mathrm{ml}$ sulfo-NHS-LC-Biotin (Pierce) in $\mathrm{PBS} / \mathrm{CM}$, and then washed for $10 \mathrm{~min}$ in $50 \mathrm{mM} \mathrm{NH}_{4} \mathrm{Cl}$ in PBS/CM. Cells were then lysed in complete Lysis buffer (1\% Triton X-100, $20 \mathrm{~mm}$ Tris, $150 \mathrm{~mm} \mathrm{NaCl}, 5 \mathrm{~mm}$ EDTA pH 8.0) and an aliquot was kept for "input." Remaining sample was incubated for $2 \mathrm{~h}$ with Immobilized Neutravidin on Agarose (Pierce). Beads were washed at least three times and resuspended in Laemmli buffer before separation by SDS-PAGE and Western blotting analysis (see below) using anti-HA (\#s.c.-805, Santa Cruz Biotechnology) primary antibodies (1:1000) and HRP-conjugated antirabbit secondary antibodies (1:5000).

Animals and drug treatments. For all experiments 8- to 10-week-old male mice were used, housed 4-5 per cage with ad libitum access to food and water. C57BL/6 mice (Charles River Laboratories) were used for ISH experiments. HTR4 BAC transgenic mice (ES88, www.gensat.org) were backcrossed to C57BL/6 strain and used for immunohistochemistry experiments. For membrane fractionation, ligand binding, and behavioral experiments, p11 knock-out and wild-type (WT) littermate controls were used (Svenningsson et al., 2006). For behavioral experiments, RS67333 (1 mg/kg, i.p.; Tocris Bioscience) or saline vehicle was delivered via intraperitoneal injection once daily for 3-5 d. The affinity of RS67333 for 5 -HTR4 binding sites $(\mathrm{pKi}=8.7)$ is greater than that for other serotonin, dopamine, and muscarinic receptors $(\mathrm{pKi}<6)$ including 5 -HT1 receptor subtypes (Eglen et al., 1995b). GR125487 (10 mg/kg, i.p., Tocris Bioscience) or saline vehicle was injected $20 \mathrm{~min}$ before RS67333 or vehicle injections. EMDT ( $5 \mathrm{mg} / \mathrm{kg}$, i.p., Eli Lilly and Company) was injected $30 \mathrm{~min}$ before testing as has been described previously (Svenningsson et al., 2007). Animal use and procedures were in accordance with the National Institutes of Health guidelines and approved by the Institutional Animal Care and Use Committees.

Primary neuronal cultures. Mixed cortical primary neuronal cultures were derived from embryonic day 18 timed pregnant female p11 KO or WT mice. After trituration with a glass pipet, cells were seeded in 12-well plates coated with poly-L-lysine $\left(1.5 \times 10^{6} \mathrm{cells} / \mathrm{ml}\right)$. Neurons were grown in neurobasal medium supplemented with $0.5 \mathrm{~mm}$ L-glutamine, $2 \%$ B27, and $1 \%$ N2. Fluorodeoxyuridine (10 $\mu \mathrm{M}$, Sigma) was added to inhibit the proliferation of non-neuronal cells; $75 \%$ of conditioned medium (CM) was replaced with fresh medium $24 \mathrm{~h}$ after seeding. Fifty percent was replaced at $2 \mathrm{~d}$ in vitro (DIV). Cells were used at 5-7 DIV.

Sample preparation and Western blotting. COS7 cells and primary neuronal cultures were rinsed in ice-cold PBS, lysed (PBS with 2 mM EDTA, 2 mM EGTA, 1\% Triton X100, 0.5\% SDS), sonicated, and centrifuged before use. Brain lysates were fractionated by centrifugation. Briefly, hippocampus was rapidly dissected, homogenized on ice $(0.32 \mathrm{M}$ sucrose, 1 mM EDTA, $0.25 \mathrm{~mm} \mathrm{DTT})$ and centrifuged $\left(1000 \times g, 10 \mathrm{~min}, 4^{\circ} \mathrm{C}\right)$. The pellet was resuspended in homogenization buffer and centrifuged again $\left(40,000 \times \mathrm{g}, 20 \mathrm{~min}, 4^{\circ} \mathrm{C}\right)$. The membrane fraction (pellet) was resuspended and sonicated in $1 \%$ SDS. Protein concentrations were measured by BCA assay (Pierce) according to manufacturer's instruction. All samples were separated on $10 \%$ Tris-glycine gels or $16 \%$ Tricine gels (Invitrogen), transferred to PVDF membrane, blocked in 5\% nonfat dry milk in Tris-buffered saline plus $0.1 \%$ Tween 20 (TBST), and incubated with primary antibodies diluted in milk overnight at $4{ }^{\circ} \mathrm{C}$ (anti-5HTR4 1:500, AbCam; anti-S100A10, R\&D Systems). Membranes were washed in TBST and incubated with HRP-conjugated secondary antibodies for $1 \mathrm{~h}$ at $4^{\circ} \mathrm{C}$ (rabbit anti-goat 1:5000; goat anti-rabbit 1:5000). Blots were incubated in ECL reagent (Perkin-Elmer), exposed to film (Kodak), and optical densities were quantified using NIH ImageJ software. For detection of MAPK, primary antibodies (rabbit anti-p44/42 MAPK, mouse anti-phosphorylated p44/42 MAPK, 1:1000, Cell Signaling Technology) were applied in Odyssey blocking buffer (Li-Cor Biosciences) overnight at $4^{\circ} \mathrm{C}$. Secondary antibodies used were 800 -anti-mouse and 680 -antirabbit (1:5000, Li-Cor Biosciences), and blots were scanned and analyzed using the Odyssey scanner (Li-Cor Biosciences).

Ligand binding. For in vitro ligand binding, membrane fractionation of hippocampus, striatum, or cortex was performed as described above. Membrane fraction (pellet) was washed $2 \times$ in binding buffer $(50 \mathrm{~mm}$ Tris $\mathrm{pH} 7.5,0.9 \% \mathrm{NaCl}$, proteinase inhibitors). Protein concentration was measured by BCA assay according to manufacturer's instructions (Pierce). $200 \mu \mathrm{g}$ of protein was incubated for $1 \mathrm{~h}$ at room temperature with $0.1 \mathrm{~nm}\left[{ }^{3} \mathrm{H}\right] \mathrm{GR} 113808$. Samples were filtered through GF/B filter, washed $3 \times$ with ice-cold binding buffer, $1 \times$ ice-cold distilled water, and air-dried overnight. Filters were moved to scintillation fluid and $\left[{ }^{3} \mathrm{H}\right]$ was counted in a scintillation counter.

In situ ligand binding was performed as described previously (Svenningsson et al., 2006). Fresh frozen $12 \mu \mathrm{m}$ coronal sections were incubated for $30 \mathrm{~min}$ at $37^{\circ} \mathrm{C}$ in $0.1 \mathrm{~nm}\left[{ }^{3} \mathrm{H}\right] \mathrm{GR} 113808$ (GE Healthcare; specific activity, $83 \mathrm{Ci} / \mathrm{mmol}$ ), $50 \mathrm{~mm}$ HEPES, $\mathrm{pH}$ 7.4, $10 \mu \mathrm{M}$ pargyline and $0.01 \%$ ascorbic acid. Nonspecific binding was determined on consecutive sections incubated in the presence of $10 \mu \mathrm{M} 5-\mathrm{HT}$. The slides were washed $2 \times 10 \mathrm{~s}$ in ice-cold binding buffer, dipped in deionized water, dried, and exposed to Kodak MR film for 6 months at $4^{\circ} \mathrm{C}$. Sections from all genotypes were processed together to obtain corresponding radiograms on the same film.

cAMP assay. cAMP production was measured using the cAMP enzyme immunoassay kit (Sigma) according to the manufacturer's instructions.

In situ hybridization. Fresh frozen $14 \mu \mathrm{m}$ coronal sections were slide mounted, fixed, dehydrated, and allowed to air dry. Then sections were rehydrated, acetylated, dehydrated and air dried again. For mRNA colocalization studies, double fluorescent in situ hybridization (ISH) was performed as described (Hommel et al., 2006). DIG- and fluoresceinlabeled riboprobes were transcribed in vitro using RNA labeling kit (Roche) from gene-specific PCR products. Sections were incubated in hybridization mixture $(50 \%$ formamide, $5 \times$ SSC, $5 \times$ Denhardts, 250 $\mu \mathrm{g} / \mathrm{ml}$ yeast RNA, $0.5 \mathrm{mg} / \mathrm{ml}$ salmon testis DNA, $200-300 \mathrm{ng} / \mathrm{ml}$ RNA probe) at $60^{\circ} \mathrm{C}$ overnight in a humidified chamber. Detection was performed exactly as described previously.

Immunohistochemistry. Mice were transcardially perfused with PBS followed by $4 \%$ paraformaldehyde. $40-\mu \mathrm{m}$-thick sections were cut on a cryostat, and every 12th section was sampled for free-floating immuno- 


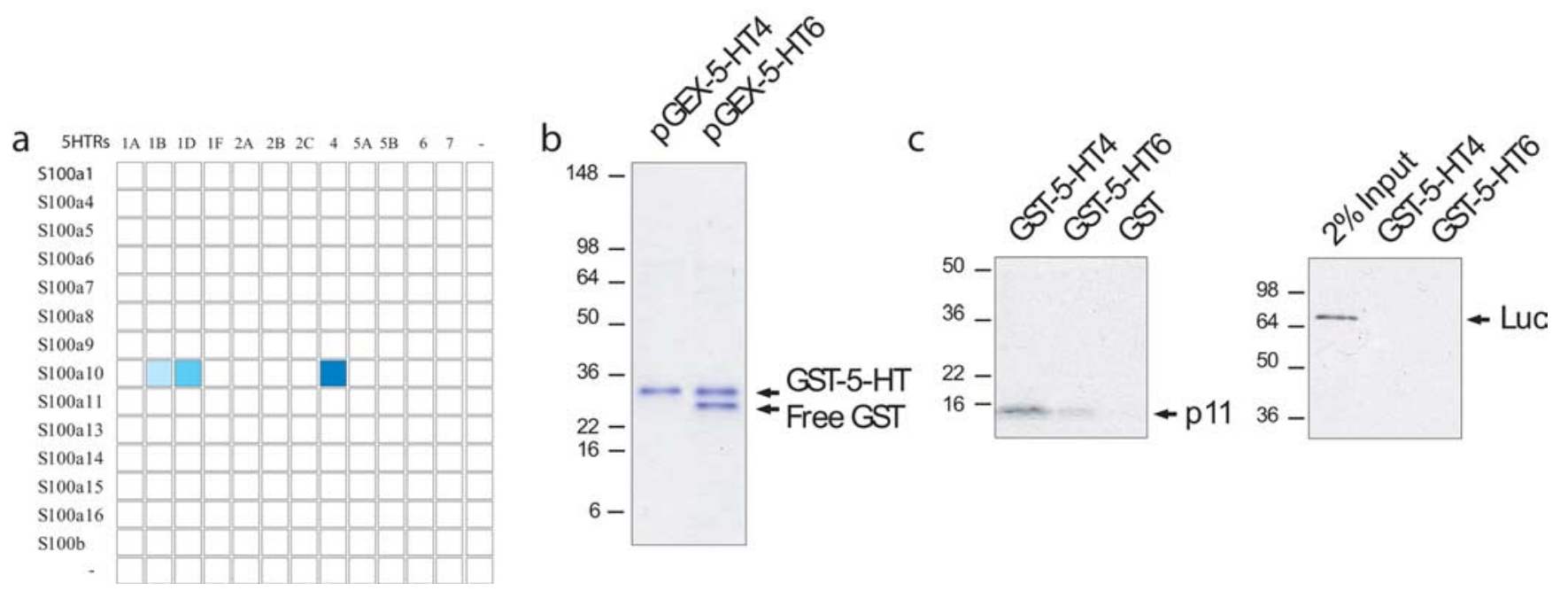

Figure 1. p11 is the only S100 protein interacting with serotonin receptors (5-HTR1B, 5-HTR1D, and 5-HTR4). $\boldsymbol{a}$, Using a yeast-based screening system, full-length S100 proteins were analyzed for interactions with the third intracellular loops of serotonin receptors. Blue boxes indicate positive interactions, darker blue representing stronger interaction. $\boldsymbol{b}, \boldsymbol{c}$, GST-pulldown assay confirmed the interaction between p11 and 5-HTR4 proteins. GST-5-HT4 or GST-5-HT6 (the entire intracellular loop 3 fused to the GST domain) were expressed in E. coli, purified, and stained with Coomassie Blue in (b). c, In vitro translated full-length p11 (left) or luciferase (right) was incubated with immobilized GST-fusion proteins (GST as negative control and GST-5-HT6 as specificity control). Retained radio-labeled products were analyzed by SDS-PAGE. 2\% input used for luciferase pull-down is shown. Size markers are indicated on the left.

histochemistry. Sections were washed with PBS, blocked in PBST plus $2 \%$ serum for $30 \mathrm{~min}$, and incubated with primary antibodies overnight at $4^{\circ} \mathrm{C}$ : rabbit anti-green fluorescent protein (GFP) 1:10,000 (AbCam); goat anti-S100A10 1:200 (R\&D Systems); and/or 1:200 chicken antiS100A10 (AbCam). Sections were washed in PBS and incubated with secondary antibodies: 1:200 alexafluor 488-donkey anti-mouse; 546donkey anti-goat; or 488-goat anti-mouse; 546-goat anti-chicken (Invitrogen) in PBS for $1 \mathrm{~h}$ at $4^{\circ} \mathrm{C}$. Then sections were washed and mounted. Images were taken on a confocal microscope (LSM510, Zeiss).

Behavioral assays. The tail suspension test (TST) was performed as described previously (Steru et al., 1985; Li et al., 2001; Mayorga et al., 2001). Mice were individually suspended by the tail from a horizontal bar (distance from floor was $35 \mathrm{~cm}$ ) using adhesive tape (distance from tip of tail was $2 \mathrm{~cm}$ ). A 6 min test session was videotaped and time spent immobile was scored by a blind observer.

For the forced swim test (FST), mice were placed in a glass cylinder (16 $\mathrm{cm}$ diameter, $25 \mathrm{~cm}$ height) filled with water $\left(23-25^{\circ} \mathrm{C}\right)$ to a height of 20 $\mathrm{cm}$. Immobility, defined as the absence of all motions except those required to keep the mouse's head above water, was scored every $5 \mathrm{~s}$ for the duration of the test (6 min) and expressed as a percentage of total time.

Elevated Plus Maze (EPM) was performed exactly as described previously (Kindlundh-Högberg et al., 2008).

Open-field behavior was recorded in four identical square open-field arenas $(50 \times 50 \times 22.5 \mathrm{~cm})$ equipped with two rows of infrared photocells placed 20 and $50 \mathrm{~mm}$ above the floor, spaced $31 \mathrm{~mm}$ apart. The last photocell in a row was spaced $17.5 \mathrm{~mm}$ from the wall. Photocell beam interruptions were collected on a computer. The variables recorded were as follows: total (or horizontal) activity (i. e. all interruptions of photobeams in the lower rows); locomotor (ambulatory) activity (i. e. successive interruptions of photocells in the lower rows); rearing (i. e. all interruptions of photobeams in the upper rows); and peripheral activity (i. e. interruptions of photobeams spaced $17.5 \mathrm{~mm}$ from the wall in the lower rows). Locomotor activity and peripheral activity (thigmotaxis) are presented as percentage of total activity.

Statistical analysis. Unless otherwise noted, all comparisons were made by Student's $t$ test. Statistical significance was set at $p<0.05$.

\section{Results}

p11 (S100A10) was identified in a yeast two-hybrid screen as a binding partner of the serotonin 5-HTR1B (Svenningsson et al., 2006). Since $\mathrm{p} 11$ is a member of a large family of $S 100$ proteins (Heizmann et al., 2002) and 5-HTR1B is one of at least 14 sub- types of 5-HT receptors expressed in the mouse brain, we determined the specificity of this interaction. We screened clones of 15 full-length S100 proteins and the third intracellular loops of 12 5-HTRs in yeast (see Materials and Methods). The third cytoplasmic loop was chosen because it is a domain that defines the receptor subtype and is the region most likely to couple to effector proteins (Attwood, 2001). This was also the region of 5-HTR1B used in the original yeast two-hybrid screen (Svenningsson et al., 2006). p11 was the only S100 protein found to interact with any of the 5-HTRs. In addition to 5-HTR1B, we found that p11 also interacts with 5-HTR4 and 5-HTR1D (Fig. 1a). We also screened for interactions between $\mathrm{p} 11$ and the third intracellular loops of noradrenergic receptors (ADR $\alpha 1 \mathrm{~A}, \mathrm{ADR} \alpha 1 \mathrm{~B}, \mathrm{ADR} \alpha 1 \mathrm{D}$, $\operatorname{ADR} \alpha 2 \mathrm{~A}, \mathrm{ADR} \alpha 2 \mathrm{~B}, \operatorname{ADR} \alpha 2 \mathrm{C}, \operatorname{ADR} \beta 1, \operatorname{ADR} \beta 2, \operatorname{ADR} \beta 3)$; no additional interactions were found (data not shown). The interaction between p11 and 5-HTR4 was confirmed in a GSTpulldown experiment using recombinant $\mathrm{p} 11$ and 5-HTR4 proteins (Fig. $1 b, c)$. We pursued the relationship between 11 and 5 -HTR4 because this was the strongest interaction identified by the screen.

If the p11/5-HTR4 interaction is physiologically relevant, the two should be coexpressed in neurons in the mouse brain. At the mRNA level, autoradiographic detection of 5-HTR4 and p11 by ISH revealed that both mRNAs are coexpressed in the cortex, striatum, amygdala and hippocampus (Magdaleno et al., 2006), brain regions that are relevant to major depression and the actions of antidepressants. Since such autoradiograms cannot show colocalization at a cellular level, we used double fluorescent ISH to demonstrate colocalization of p11 and 5-HTR4 mRNAs in individual cells (Fig. 2). Quantification of colabeled cells indicated that $90-100 \%$ of cells expressing 5 -HTR 4 also express p 11 in all brain regions examined (Table 1).

Using BAC transgenic mice that express enhanced GFP (eGFP) under the 5-HTR4 promoter (Gong et al., 2003) (www.gensat.org), we showed that p11 protein was expressed in the cells expressing 5-HTR4. Immunohistochemical detection of p11 and eGFP revealed colocalization in cortex, striatum, amygdala, dentate gyrus and CA1 pyramidal cell layers (Fig. 3). Immunostaining was also 


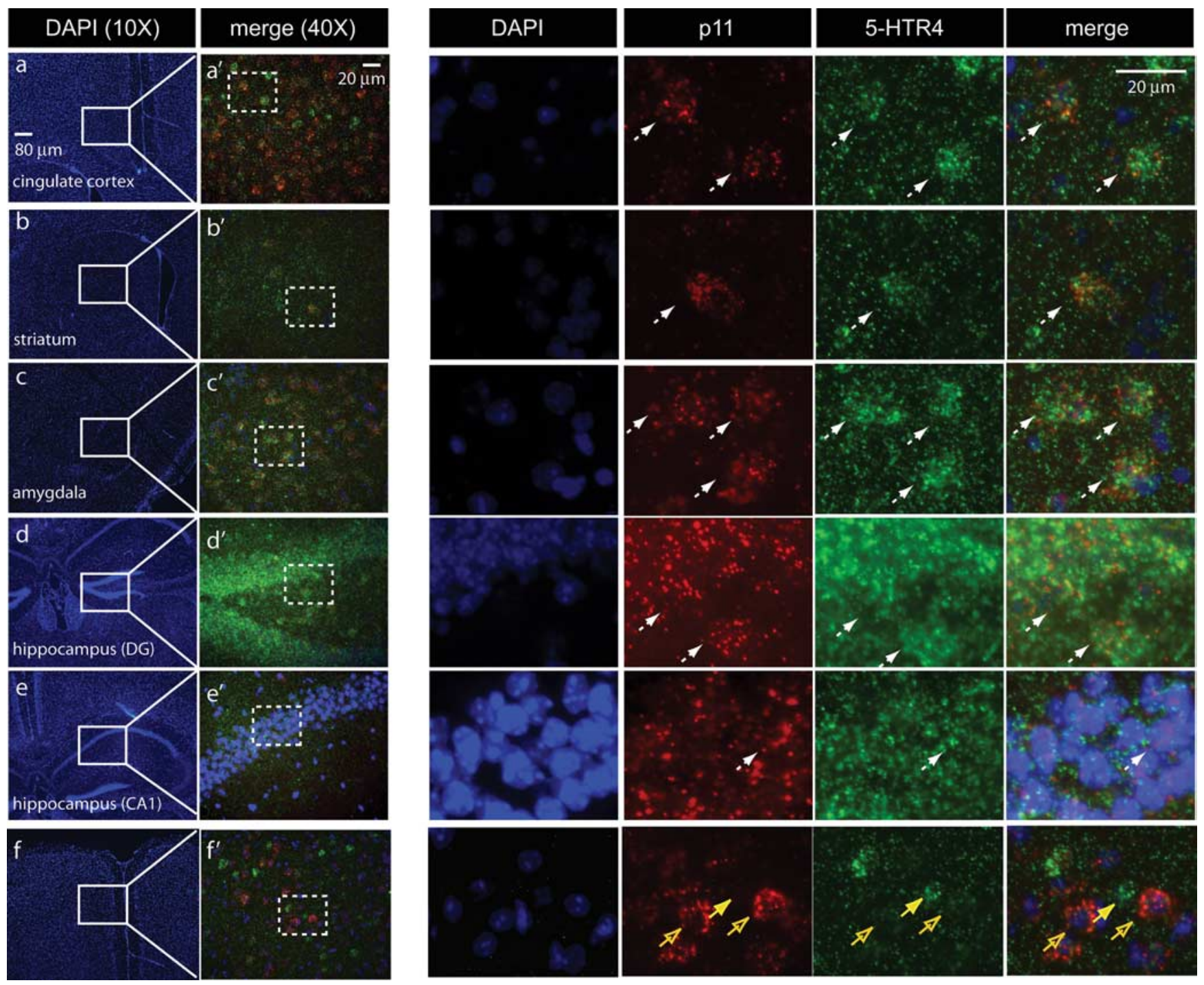

Figure 2. Colocalization of 11 and 5 -HTR4 mRNA in depression-related brain regions. mRNAs were detected in wild-type mice by double fluorescent in situ hybridization (FISH) using dig-labeled p11 (red) and fluorescein-labeled 5-HTR4 (green) probes with DAPI (blue) nuclear counterstain. $\boldsymbol{a}-\boldsymbol{f}$, Low magnification images stained with DAPI illustrate brain regions included in analysis: (a) cortex, $(\boldsymbol{b})$ striatum, $(\boldsymbol{c})$ amygdala, $(\boldsymbol{d})$ dentate gyrus of hippocampus, and $(\boldsymbol{e})$ CA1 pyramidal cell layer of hippocampus. $(\boldsymbol{f})$ is an example in which some p11-positive cells do not express 5-HTR4. $\boldsymbol{a}^{\prime}-\boldsymbol{f}^{\prime}$ correspond to the field included in the white boxes in $\boldsymbol{a}-\boldsymbol{f}$. Dotted boxes in $\boldsymbol{a}^{\prime}-\boldsymbol{f}^{\prime}$ are magnified to the right. White arrows indicate cells expressing both p11 (red) and 5-HTR4 (green) mRNAs against the DAPI (blue) counterstain. Yellow arrow indicates one 5-HTR4 cell that does not coexpress p11, and open yellow arrows indicate cells expressing p11, but not 5-HTR4.

performed using a different polyclonal p11 antibody (see Materials and Methods) and yielded identical results (data not shown).

\section{p11 increases 5-HTR4}

surface expression

p11 increases surface expression of 5-HTR1B and facilitates signaling through this receptor in cell culture systems (Svenningsson et al., 2006). Here we investigated whether p11 has similar effects on 5-HTR4. Immunohistochemical detection of 5-HTR4 and p11 illustrated colocalization at the cell surface of transfected COS7 cells expressing both 5-HTR4 and p11 (Fig. 4a). When p11 is not cotransfected with 5-HTR4, 5-HTR4 is localized at the cell surface (Fig. $4 b$ ), likely due to the relatively low levels of p11 found endogenously in COS7 cells. However, when p11 is transfected in the absence of 5-HTR4 (Fig. 4c), p11 expression is cytoplasmic. To quantitatively determine if 11 increased 5-HTR4 localization
Table 1. Percentage of 5-HTR4-positive cells coexpressing p11 mRNA

\begin{tabular}{lcccc}
\hline Brain region & Mouse 1 & Mouse 2 & Mouse 3 & Average (SEM) \\
\hline Cortex & 89.8 & 96.1 & 98 & $94.6(2.5)$ \\
Striatum & 89.3 & 96.6 & 93.3 & $93.0(2.1)$ \\
Amygdala & 91.4 & 92 & 91 & $91.5(0.3)$ \\
Hippocampus (DG) & 100 & 100 & 100 & $100(0)$ \\
Hippocampus (CA1) & 98.9 & 98 & 98 & $98.3(0.3)$
\end{tabular}

Quantification of images of the type shown in Figure 2 demonstrated that $90-100 \%$ of cells expressing 5-HTR4 mRNA also express p 11 mRNA. Three $557 \mathrm{BL} / 6$ male mice were used for this analysis. For each brain region, five images per mouse were analyzed ( $150-200$ cells per mouse per region).

at the plasma membrane, we used a biotinylation assay. Results show that cotransfection of p11 with 5-HTR4 significantly increased the amount of 5-HTR4 at the cell surface compared with cells transfected with 5-HTR4 only (Fig. 4d).

We also determined whether surface localization of 5-HTR4 receptors was affected by 11 in vivo by fractionating brain lysates from adult p11 knock-out or wild-type mice. Hippocampus from p11 knock-out or wild-type mice was fractionated by centrifugation. Total hippocampus homogenate or plasma membrane en- 


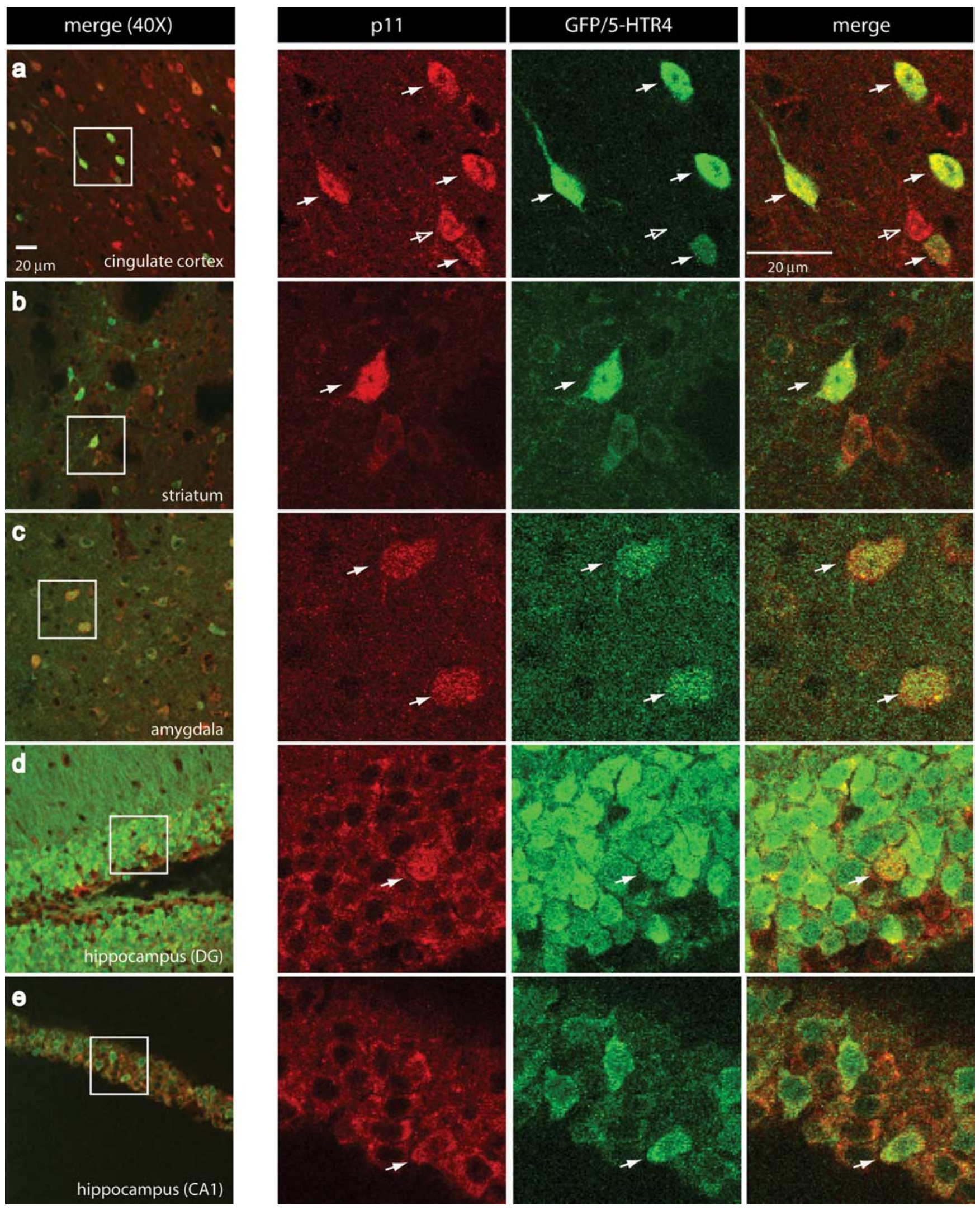

Figure 3. p11 protein colocalizes with 5-HTR4. a-e, Immunohistochemistry was performed using antibodies recognizing p11 (red) or GFP (green) in brain sections from BAC transgenic mice overexpressing eGFP under the 5-HTR4 promoter. Confocal micrographs taken from $(\boldsymbol{a})$ cingulate cortex, $(\boldsymbol{b})$ striatum, $(\boldsymbol{c})$ amygdala, (d) dentate gyrus of the hippocampus and (e) CA1 pyramidal cell layer of the hippocampus at $40 \times$ magnification. White boxes in $\boldsymbol{a}$ - $\boldsymbol{e}$ are enlarged to the right to illustrate single cells (white arrows) expressing both p11 (red) and GFP/5-HT4 (green). 0 pen arrow in $a$ points to p11-positive cell that does not express GFP. 
riched fractions were analyzed by Western blotting analysis. Results showed that p11 knock-out mice had 20\% less 5-HTR4 surface expression compared with wild-type littermate controls (Fig. 4e). There was no effect of genotype on total 5-HTR4 protein level. We also analyzed surface expression of 5-HTR 4 by ligand binding experiments. These were performed on membrane fractions of cortex, hippocampus and striatum using $\left[{ }^{3} \mathrm{H}\right] \mathrm{GR} 113808$. In the hippocampus, a region in which there is an abundance of 5-HTR 4 receptors, a $25 \%$ reduction in ligand binding was detected in p11 KO mice compared with WT littermates $(p<0.05, n=3)$. In striatum and cortex, we detected a $20 \%$ and $12 \%$ reduction, respectively, but these effects did not reach statistical significance $(p=0.06, p=0.13$, respectively; $n=3$ ) (Fig. $4 f$ ). In addition, ligand binding autoradiography using $\left[{ }^{3} \mathrm{H}\right] \mathrm{GR} 113808$ was performed in brain sections (Fig. $4 g$ ). Quantification in the striatum of p11 KO and WT mice revealed a $22 \%$ reduction of $\left[{ }^{3} \mathrm{H}\right]$ GR 113808 binding in p11 KO mice compared with WT littermates (KO: $77.8 \pm 3.9 \%$; WT: $100 \pm$ $7.9 \%$, mean \pm SEM, $p<0.05)$.

p11 increases signaling through 5-HTR4 5-HTR4 is a $\mathrm{G}_{\alpha \mathrm{s}}$-coupled receptor that increases cAMP production upon receptor activation. Therefore, we measured cAMP levels in transfected COS-7 cells stimulated with 5-HT. Cotransfection of p11 with 5-HTR4 potentiated cAMP production above cells that overexpressed 5-HTR4 alone (Fig. 5a). Furthermore, cotransfection of p11 with 5-HTR6, a receptor with which there is no interaction, had no effect on cAMP production (Fig. $5 a$ ). To test whether p11 affects 5-HTR4 mediated cAMP production in neurons, we stimulated primary cultures from p11 WT and KO mice with 5-HTR4 agonist RS67333. The response to RS67333 stimulation in p11 KO cultures was reduced by $>50 \%$ compared with WT controls (Fig. 5b).

Activation of 5-HTR4 also increases phosphorylation of MAPK (Norum et al., 2003; Barthet et al., 2007). Cotransfecting p11 and 5-HTR4 in COS7 cells significantly increased serotonin activation of phosphorylation of both p42 and p44 MAPK compared with cells overexpressing 5-HTR4 alone (Fig. 5c). There were no significant differences in MAPK phosphorylation in the absence of 5-HT stimulation (data not shown). Coexpression of p11 and 5-HTR6, which also activates MAPK, had no effect on MAPK phosphorylation (Fig. 5c).

To determine whether similar results are observed in neurons, we applied the 5-HTR4 partial agonist RS67333 to primary cortical neurons derived from $\mathrm{p} 11$ knock-out mice or wild-type controls. RS67333 increased phosphorylation of MAPK in wild-type neurons, but not in neurons isolated from p11 knock-out mice (Fig. $5 d$ ).

Together, these data from cell lines, primary neuronal cul-
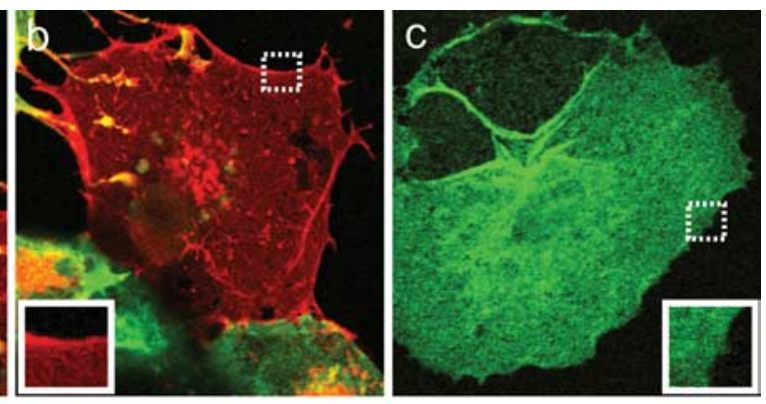

e
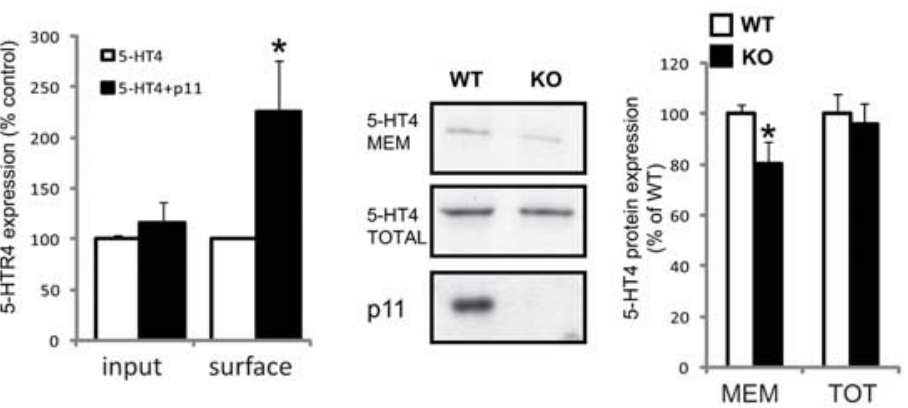

g

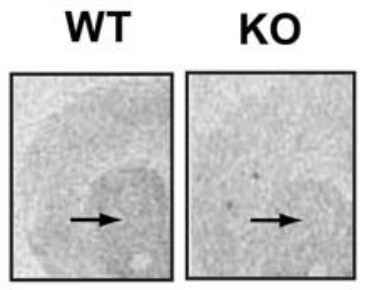

STR

Figure 4. $\quad$ p11 increases 5-HTR4 expression at the plasma membrane. $\boldsymbol{a}-\boldsymbol{c}$, COS7 cells were transfected with (a) p11 (green) and 5-HTR4 (red), (b) 5-HTR4 alone or (c) p11 alone. Regions included in the inset are marked by white dotted boxes. White arrow head in $\boldsymbol{a}$ indicates colocalization of p11 and 5-HTR4 at cell surface. $\boldsymbol{d}$, Biotinylation experiment from transfected COS7 cells. Data

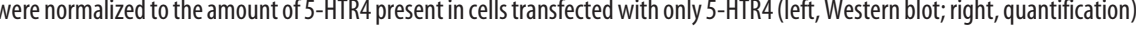
e, 5-HTR4 protein expression in plasma membrane enriched fraction versus total hippocampal lysate from p11 WT or K0 mice (left, blot; right, quantification). f, In vitro 5-HTR4 ligand binding assay using $\left[{ }^{3} \mathrm{H}\right] \mathrm{GR} 113808$ in cortex, hippocampus, and striatum of p11 K0 or WT mice. $g$, Representative image for 5-HTR4 ligand binding in striatum (arrows) of p11 WT or KO mice (for quantification, see Results). All data are presented as mean \pm SEM, ${ }^{*} p<0.05$.

tures, and adult brain lysates support the conclusion that $\mathrm{p} 11$ increases 5-HTR4 expression at the cell surface and increases 5 -HTR4 signaling.

\section{p11 is required for the behavioral antidepressant action of a 5-HTR4 agonist}

We have shown previously that $\mathrm{p} 11$ is required for the behavioral antidepressant actions of a 5-HTR1B agonist (Svenningsson et al., 2006). The 5-HTR4 partial agonist RS67333 has been shown recently to produce antidepressant-like effects in rodent models of depression (Lucas et al., 2007). To determine whether p11 is also required for the behavioral antidepressant actions of a 5-HTR4 agonist, we injected p11 knock-out and wild-type littermates with RS67333 and tested them in two classic behavioral paradigms: the tail suspension test (TST) and the forced swim test (FST). In both tests, decreased immobility was scored as an index of antidepressant activity (Cryan et al., 2002).

In confirmation of earlier studies, p11 knock-out mice given vehicle showed increased immobility in both tests. In the TST, $3 \mathrm{~d}$ of RS67333 (1 mg/kg, i.p.) significantly reduced im- 
a

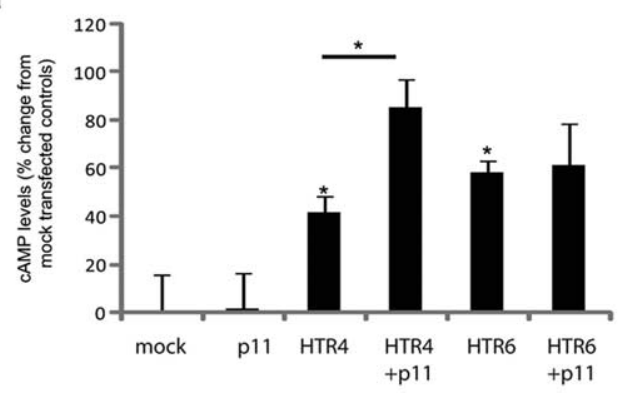

C

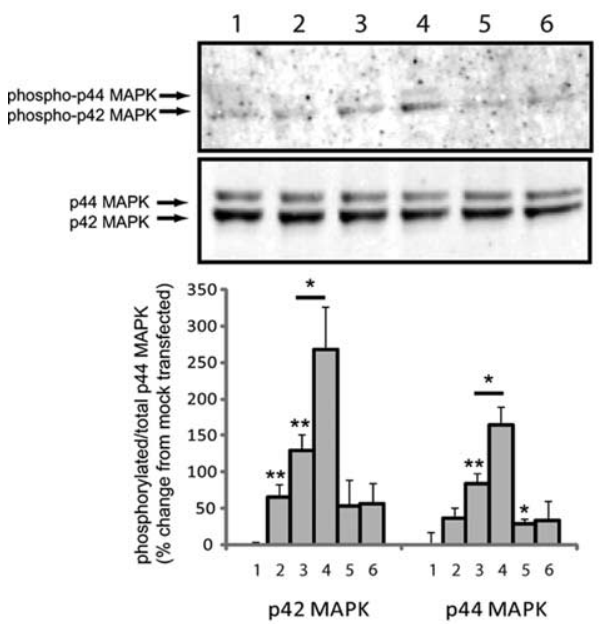

b
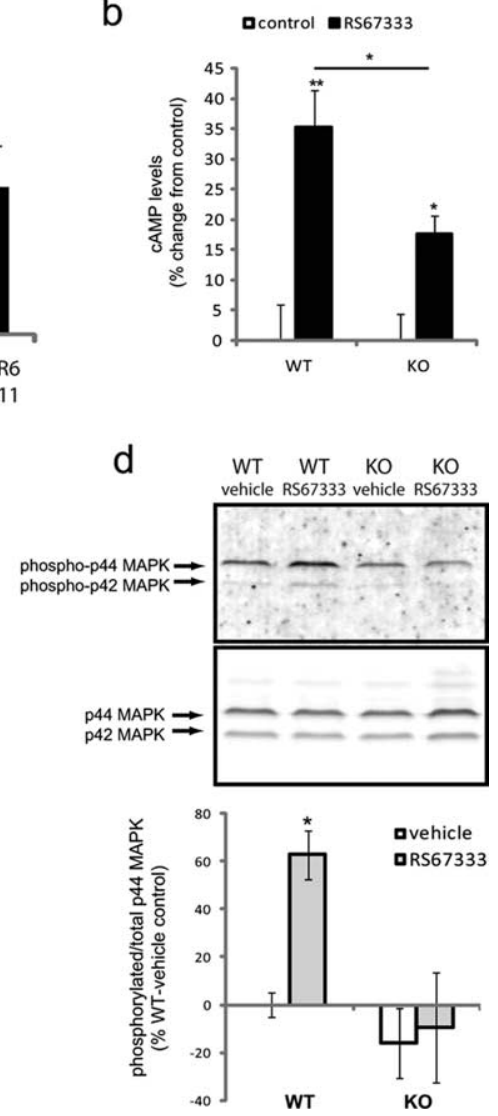

Figure 5. p11 facilitates signaling through 5-HTR4. $\boldsymbol{a}$, Effect of 5-HT (1 $\mu \mathrm{M})$ on cAMP levels in COS7 cells transfected with p11, 5-HTR4, p11 and 5-HTR4, 5-HTR6, or p11 and 5-HTR6. $\boldsymbol{b}$, Effect of stimulation with 5-HTR4 partial agonist RS67333 $(10 \mu \mathrm{m})$ in primary neuronal cultures derived from $\mathrm{p} 11 \mathrm{WT}$ or K0 mice. Increase in cAMP levels in response to RS67333 was blunted by $>50 \%$ in p11 $\mathrm{KO}$ cultures compared with WT. c, Quantification and representative image from Western blotting analysis of transfected COS7 cells in the presence of serotonin $(1 \mu \mathrm{m})$. Cotransfection of p11 with 5-HTR4 increased phosphorylation of MAPK compared with 5 -HTR4 alone $(1=$ mock, $2=p 11,3=5$-HTR4, $4=5$-HTR4 and p11, $5=5$-HTR6, and $6=5$-HTR6 and p11).d, Primary cortical neuronal cultures derived from $\mathrm{p} 11$ WT or K0 mice stimulated with RS67333. RS67333 increased phosphorylation of MAPK in WT, but not in KO cultures. All data are presented as mean \pm SEM, ${ }^{*} p<0.05,{ }^{* *} p<0.01$.

mobility in wild-type mice, but had no significant effect in p11 knock-out mice (Fig. $6 a$ ). In contrast, EMDT ( $5 \mathrm{mg} / \mathrm{kg}$, i.p.) stimulation of 5-HTR6, a 5-HT receptor that does not interact with p11, produced a significant antidepressant-like effect in both WT and KO mice (Fig. 6a) (Svenningsson et al., 2007). To confirm that the effect of RS67333 was due to activation of 5-HTR4, we injected WT mice with GR125487 $(10 \mathrm{mg} / \mathrm{kg}$, i.p.), a 5-HTR4 antagonist, in the presence or absence of RS67333. Pretreatment with the 5-HTR4 antagonist abolished the effect of RS67333 on immobility in the TST (Fig. 6b). In the FST, RS67333 significantly reduced immobility in wildtype mice compared with saline-injected controls (35.6 \pm 3.6 vs $23.9 \pm 1.8$ counts \pm SEM, $p<0.05)$, but had no effect in p11 knock-out mice ( $47.4 \pm 4.2$ vs $44.0 \pm 2.8)$ (Fig. 6c). Thigmotaxis is a measure of anxiety that is reduced by treatment with antidepressants. RS67333 significantly reduced thigmotaxis in wild-type controls $(0.92 \pm 0.036$ vs $0.73 \pm 0.076)$, but had no effect in p11 knock-out mice $(0.88 \pm 0.025$ vs $0.88 \pm$ 0.038 peripheral/total horizontal activity \pm SEM) (Fig. $6 d$ ). We also tested mice in the Elevated Plus Maze (EPM), in which time spent in open versus closed arms of the maze is a well established measure of anxiety. In WT mice, RS67333 increased time spent in the open arms of the maze (saline: $5.4 \pm$ 1.3 vs RS67333: $8.8 \pm 2.2 \mathrm{~s} \pm$ SEM), but this effect did not

reach statistical significance $(p=0.14$, $n=16$ per group). There was no such trend in p11 KO mice treated with RS67333 (saline: $5.2 \pm 1.1$ vs RS67333: $4.1 \pm 1.0 \mathrm{~s} \pm \mathrm{SEM}, p=0.24, n=22$ or 17 , respectively). Since all of these behaviors rely on locomotor responses, we also measured locomotor activity in an open field. There were no significant differences between groups (WT-vehicle: $707 \pm$ 104; WT-RS67333: $894 \pm 140$; KO-vehicle: $840 \pm 78$; KO-RS67333: $751 \pm 85$ activity counts \pm SEM).

\section{Discussion}

We previously identified p11 as an antidepressant target and regulator of depressive-like states (Svenningsson et al., 2006). In rodent behavioral models, p11 knock-out mice display a depression-like phenotype and are resistant to antidepressant treatment, while over-expression of p11 mimics the action of antidepressants. The effects of p1 1 are mediated, in part, by interactions with the serotonin receptor $1 \mathrm{~B}$ (5-HTR1B). p1 1 acts as an adaptor protein for 5-HTR1B to facilitate surface expression and downstream signaling. In addition, p11 knock-out mice are less responsive to the 5-HT1B agonist anpirtoline in behavioral assays.

In the present study, we identified a strong interaction between $\mathrm{p} 11$ and another serotonin receptor: 5-HTR4. This interaction (1) is physiologically relevant, (2) facilitates signaling through 5-HTR4, and (3) plays a role in antidepressant activity.

p11 is the only S100 protein to interact with any serotonin receptor, thus revealing a high degree of specificity of the interaction between p11 and serotonin receptors. p11 has mutations in both EFhands (Heizmann et al., 2002), rendering the protein insensitive to calcium and making it a unique member of the $S 100$ protein family. This suggests that the regulation and function of $\mathrm{p} 11$ is distinct from that of other S100 proteins.

p11 interacts specifically with serotonin receptor subtypes $1 \mathrm{~B}, 1 \mathrm{D}$, and 4.5-HTR1B and 5-HTR1D are members of the type- 1 class of serotonin receptors. These receptors are negatively coupled to adenylyl cyclase, reducing cAMP production when the receptor is activated and inhibiting the excitability of the cell. It is notable that 5-HTR1B and 5-HTR1D are $62.2 \%$ identical and $77.48 \%$ homologous in sequence. These receptors have had a complicated nomenclature history due to initial pharmacological characterization of the two receptors. 5 -HTR1B in the rat/mouse is analogous to the human $5-\mathrm{HT} 1 \mathrm{D} \beta$ receptor, and the rodent 5 -HT1D receptor is called 5-HT1D $\alpha$ in the human. The functional role of 5-HTR1D in the mouse is not clear, although based on its sequence similarity to 5 -HTR $1 \mathrm{~B}$, and the fact that both are expressed on nerve terminals (Bruinvels et al., 1994), it has been speculated that they share similar functions. 5-HTR1B and 5-HTR1D localization are largely overlapping in the ro- 


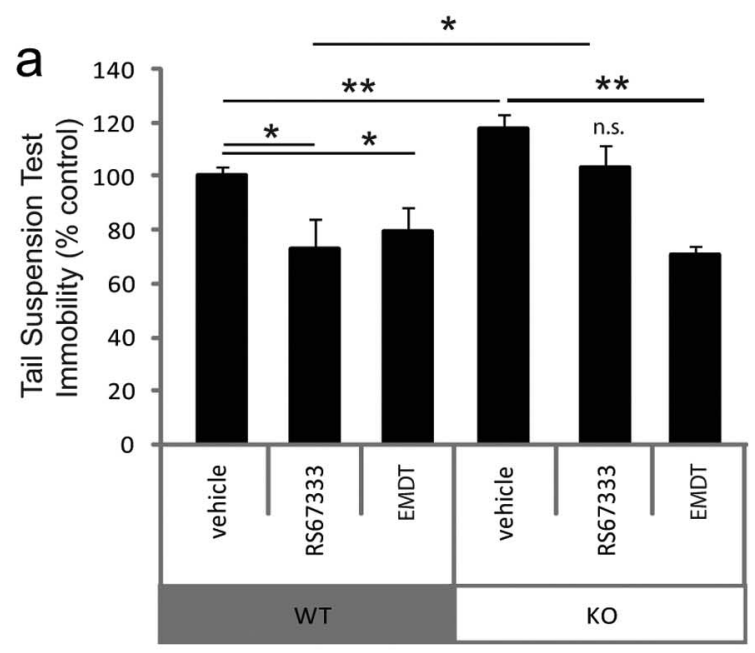

b

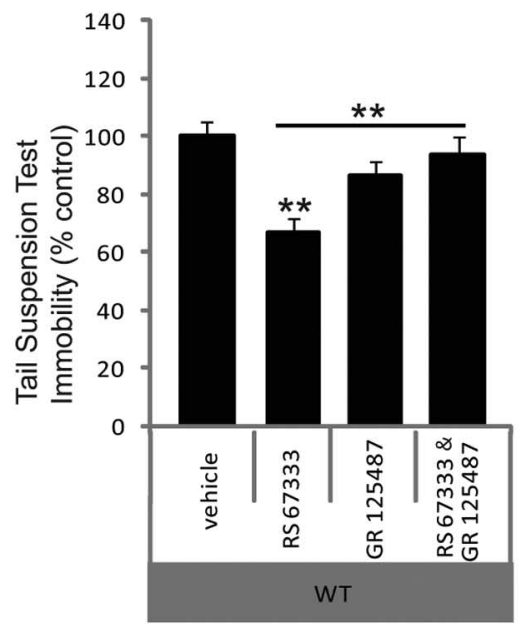

C

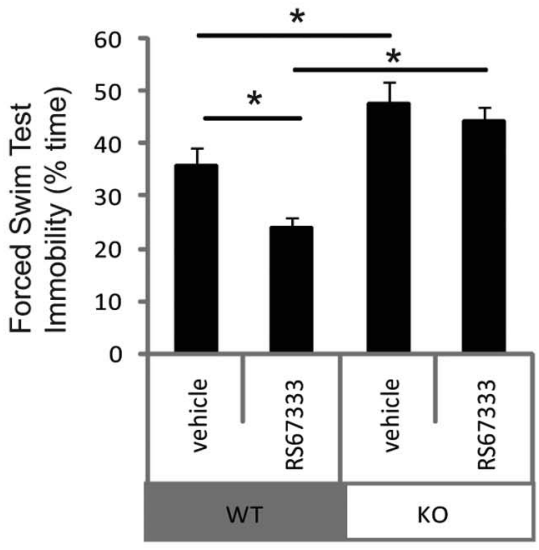

d

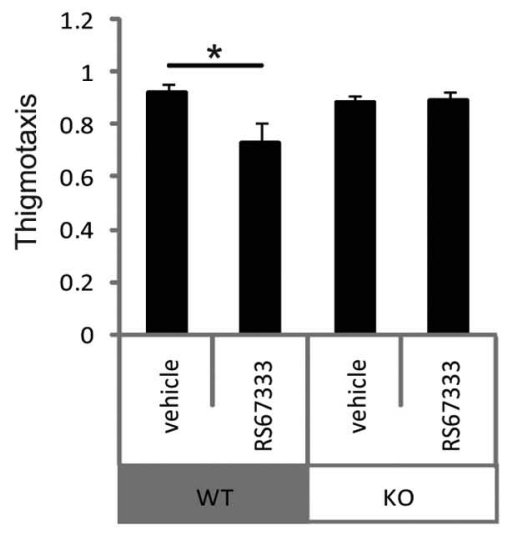

Figure 6. $p 11$ is required for the behavioral antidepressant actions of a selective 5 -HTR4 partial agonist (RS67333). p11 knock-outs and wild-type littermate controls were injected with RS67333 or saline vehicle for 3-5 d before behavioral testing. Immobility is an index of antidepressant activity in both the TST and FST. $\boldsymbol{a}, \boldsymbol{c}$, Consistent with the action of an antidepressant, RS67333 reduced time spent immobile in the TST $(\boldsymbol{a})$ and FST (c) in wild-type mice but had no effect in p11 knock-out mice. In the TST (a) EMDT, an agonist for 5 -HTR6 (a 5-HT receptor that does not interact with p11) reduced immobility in both p11 K0 and WT mice. $\boldsymbol{b}$, Treatment of WT mice with GR125487, a 5-HTR4 antagonist, blocked the antidepressant-like effect of RS67333 in the TST. $\boldsymbol{d}$, RS67333 had an anxiolytic effect in the Open Field, reducing thigmotaxis in wild-type mice, but not in p11 knock-out mice. All data are presented as means \pm SEM ${ }^{*} p<0.05,{ }^{* *} p<0.01$

dent brain, specifically in cortex and caudate/putamen (Bruinvels et al., 1994), but the 5-HT1B receptor is expressed at a higher level in the adult (Bolaños-Jiménez et al., 1997) and is also found in other brain regions, such as hippocampus, where no 5-HTR1D is expressed. Most available pharmacological agonists and antagonists of 5-HTR1D also act on 5-HTR1B. Therefore, we decided to pursue the interaction between p11 and 5-HTR4 since (1) there was a lack of pharmacological tools to study 5-HTR1D, (2) adult brain expression of 5-HTR1D is limited, and (3) the interactions with 5-HTR1B and 5-HTR1D were weaker.

5-HTR4 does not share sequence homology with 5-HTR1B/D, belonging to a different subtype of serotonin receptor. 5-HTR4 is positively coupled to adenylyl cyclase, increasing levels of cAMP when the receptor is stimulated. 5-HTR4 is highly expressed in the rodent cortex, striatum, hippocampus, septum, and amygdala (Waeber et al., 1996; Varnäs et al., 2003), and is located postsynaptically where it modulates neurotransmitter (e.g., acetylcholine) release from the postsynaptic cell (Fink and Göthert, 2007). We find that cells that express 5 -HTR4 invariably coexpress p11, in brain regions relevant to major depression, demonstrating that this interaction is physiologically relevant.

Functionally, p11 is an adaptor protein for several channels and GPCRs (Svenningsson and Greengard, 2007). The present study using transfected cells, primary neuronal cultures, and mouse brain strongly suggests that p11 regulates surface expression of 5-HTR4 as well. Cotransfection of p11 with 5-HTR4 increases cAMP production and phosphorylation of MAPK. In primary neuronal cultures derived from p11 knock-out mice, stimulation of 5-HTR4 with the agonist RS67333 failed to increase cAMP or levels of MAPK phosphorylation. Cotransfection of p11 with 5-HTR6, another class of $\mathrm{G}_{\alpha \mathrm{s}}$-coupled serotonin receptor, which does not interact with $\mathrm{p} 11$, does not affect signaling through 5-HTR6. These data provide evidence that $\mathrm{p} 11$ increases surface expression of functional 5-HTR4.

Typically, antidepressants require weeks or months of treatment before a therapeutic effect is observed, and then actions include a host of unpleasant side-effects. Therefore, the search continues for faster-acting antidepressants. Re- 
cently, 5-HTR4 was identified as a faster-acting antidepressant target (Lucas et al., 2007). Lucas et al., demonstrated that cellular and behavioral responses that require 2-3 weeks of antidepressant treatment in rodents will occur after only $1-3 \mathrm{~d}$ of treatment with a selective 5-HTR4 partial agonist (RS67333) (Eglen et al., 1995b). Here we demonstrate in three behavioral paradigms that the antidepressant activity of RS67333 is absent in p11 knock-out mice compared with wildtype littermate controls. We also show that the antidepressant effect of stimulating 5-HTR6 (Svenningsson et al., 2007), a serotonin receptor that does not interact with $\mathrm{p} 11$, is not affected in p11 KO mice. This is consistent with our cellular and biochemical findings, suggesting that there is less 5-HTR4 expression at the cell surface in the p11 KO mice. Neither genotype nor treatment had a significant effect on general locomotor activity, which is critical since all of these behaviors require locomotor responses.

Use of 5-HTR4 as a novel antidepressant target may be hampered by the fact that there is a high level of expression of these receptors in the gastrointestinal system (Tonini and Pace, 2006). Indeed, 5-HTR4 agonists are used in humans for the treatment of gastrointestinal disorders, like irritable-bowel syndrome (Tonini and Pace, 2006). In addition, 5-HTR4 receptors may mediate the appetite-related component of stress disorders (Compan et al., 2004; Jean et al., 2007).

The localization of 5-HTR4 in limbic brain regions is suggestive of emotional and cognitive functions (Eglen et al., 1995a). In addition to its role in depression-related behaviors, p11 could modulate learning and memory via its interaction with 5-HTR4.

The interactions between $\mathrm{p} 11$ and 5-HTRs are highly specific. We believe that the antidepressant activities of $\mathrm{p} 11$ are mediated by interactions with both 5-HTR1B and 5-HTR4. Therefore, it will be important to determine the relative contribution of each receptor to the antidepressant activities of p11. We hypothesize that since p11 is widely expressed in brain regions that are relevant to depression (e.g., cortex, hippocampus, striatum, amygdala, hypothalamus), 5-HTR4 could be more relevant to p11 activity in one brain region (e.g., hippocampus), whereas 5-HTR1B mediated effects could be localized to another brain region (e.g., cortex). This is based on observations that (1) there is a high level of 5-HTR4 in the hippocampus, a brain region that is strongly linked to depression and antidepressant activity (WarnerSchmidt and Duman, 2006) and (2) serotonin inhibits corticoaccumbal synaptic transmission via presynaptic 5-HT1B receptors (Muramatsu et al., 1998) in a p11-dependent way (Svenningsson et al., 2006). Alternatively, within the same brain area, p1 1 could interact with 5-HTR4 and 5-HTR1B on opposing cell types. Future studies will determine the brain region and cell-type specific effects of $\mathrm{p} 11$ and its interactions with 5-HTR1B and 5-HTR4.

There is a pressing clinical need for faster-acting antidepressants. Patients often wait weeks or months before feeling the therapeutic effects of the currently available drugs, if these treatments work at all. An understanding of the cellular mechanisms underlying the therapeutic actions of these drugs may lead to better treatments with fewer side-effects. p11 and its specific interactions with 5-HTR4 and 5-HTR1B represent a novel and promising avenue of investigation.

\section{References}

Attwood TK (2001) A compendium of specific motifs for diagnosing GPCR subtypes. Trends Pharmacol Sci 22:162-165.

Barnes NM, Sharp T (1999) A review of central 5-HT receptors and their function. Neuropharmacology 38:1083-1152.
Barthet G, Framery B, Gaven F, Pellissier L, Reiter E, Claeysen S, Bockaert J, Dumuis A (2007) 5-hydroxytryptamine 4 receptor activation of the extracellular signal-regulated kinase pathway depends on Src activation but not on G protein or beta-arrestin signaling. Mol Biol Cell 18:1979-1991.

Bolaños-Jiménez F, Choi DS, Maroteaux L (1997) Preferential expression of 5-HT1D over 5-HT1B receptors during early embryogenesis. Neuroreport 8:3655-3660.

Bruinvels AT, Landwehrmeyer B, Gustafson EL, Durkin MM, Mengod G, Branchek TA, Hoyer D, Palacios JM (1994) Localization of 5-HT1B, 5-HT1D alpha, 5-HT1E and 5-HT1F receptor messenger RNA in rodent and primate brain. Neuropharmacology 33:367-386.

Compan V, Zhou M, Grailhe R, Gazzara RA, Martin R, Gingrich J, Dumuis A, Brunner D, Bockaert J, Hen R (2004) Attenuated response to stress and novelty and hypersensitivity to seizures in 5-HT4 receptor knock-out mice. J Neurosci 24:412-419.

Cryan JF, Markou A, Lucki I (2002) Assessing antidepressant activity in rodents: recent developments and future needs. Trends Pharmacol Sci 23:238-245.

Donato R (1999) Functional roles of S100 proteins, calcium-binding proteins of the EF-hand type. Biochim Biophys Acta 1450:191-231.

Eglen RM, Wong EH, Dumuis A, Bockaert J (1995a) Central 5-HT4 receptors. Trends Pharmacol Sci 16:391-398.

Eglen RM, Bonhaus DW, Johnson LG, Leung E, Clark RD (1995b) Pharmacological characterization of two novel and potent 5-HT4 receptor agonists, RS 67333 and RS 67506, in vitro and in vivo. Br J Pharmacol 115:1387-1392.

Fink KB, Göthert M (2007) 5-HT receptor regulation of neurotransmitter release. Pharmacol Rev 59:360-417.

Gong S, Zheng C, Doughty ML, Losos K, Didkovsky N, Schambra UB, Nowak NJ, Joyner A, Leblanc G, Hatten ME, Heintz N (2003) A gene expression atlas of the central nervous system based on bacterial artificial chromosomes. Nature 425:917-925.

Grabe HJ, Ahrens N, Rose HJ, Kessler C, Freyberger HJ (2001) Neurotrophic factor S100 beta in major depression. Neuropsychobiology 44:88-90.

Heizmann CW, Fritz G, Schäfer BW (2002) S100 proteins: structure, functions and pathology. Front Biosci 7:d1356-d1368.

Hommel JD, Trinko R, Sears RM, Georgescu D, Liu ZW, Gao XB, Thurmon JJ, Marinelli M, DiLeone RJ (2006) Leptin receptor signaling in midbrain dopamine neurons regulates feeding. Neuron 51:801-810.

Jean A, Conductier G, Manrique C, Bouras C, Berta P, Hen R, Charnay Y, Bockaert J, Compan V (2007) Anorexia induced by activation of serotonin 5-HT4 receptors is mediated by increases in CART in the nucleus accumbens. Proc Natl Acad Sci U S A 104:16335-16340.

Kindlundh-Högberg AM, Zhang X, Svenningsson P (2008) S100B overexpressing mutant mice exhibit prolonged behavioural and biochemical responses towards repeated intermittent binge treatments with MDMA. Int J Neuropsychopharmacol 24:1-15.

Li X, Tizzano JP, Griffey K, Clay M, Lindstrom T, Skolnick P (2001) Antidepressant-like actions of an AMPA receptor potentiator (LY392098). Neuropharmacology 40:1028-1033.

Lucas G, Rymar VV, Du J, Mnie-Filali O, Bisgaard C, Manta S, Lambas-Senas L, Wiborg O, Haddjeri N, Piñeyro G, Sadikot AF, Debonnel G (2007) Serotonin $(4)\left(5-\mathrm{HT}_{(4)}\right)$ receptor agonists are putative antidepressants with a rapid onset of action. Neuron 55:712-725.

Magdaleno S, Jensen P, Brumwell CL, Seal A, Lehman K, Asbury A, Cheung T, Cornelius T, Batten DM, Eden C, Norland SM, Rice DS, Dosooye N, Shakya S, Mehta P, Curran T (2006) BGEM: an in situ hybridization database of gene expression in the embryonic and adult mouse nervous system. PLoS Biol 4:e86.

Mayorga AJ, Dalvi A, Page ME, Zimov-Levinson S, Hen R, Lucki I (2001) Antidepressant-like behavioral effects in 5-hydroxytryptamine(1A) and 5-hydroxytryptamine(1B) receptor mutant mice. J Pharmacol Exp Ther 298:1101-1107.

Muramatsu M, Lapiz MD, Tanaka E, Grenhoff J (1998) Serotonin inhibits synaptic glutamate currents in rat nucleus accumbens neurons via presynaptic 5-HT1B receptors. Eur J Neurosci 10:2371-2379.

Norum JH, Hart K, Levy FO (2003) Ras-dependent ERK activation by the human G(s)-coupled serotonin receptors 5-HT4(b) and 5-HT7(a). J Biol Chem 278:3098-3104.

Rothermundt M, Arolt V, Wiesmann M, Missler U, Peters M, Rudolf S, 
Kirchner H (2001) S-100B is increased in melancholic but not in nonmelancholic major depression. J Affect Disord 66:89-93.

Steru L, Chermat R, Thierry B, Simon P (1985) The tail suspension test: a new method for screening antidepressants in mice. Psychopharmacology 85:367-370.

Svenningsson P, Greengard P (2007) p11 (S100A10)-an inducible adaptor protein that modulates neuronal functions. Curr Opin Pharmacol 7:27-32.

Svenningsson P, Chergui K, Rachleff I, Flajolet M, Zhang X, El Yacoubi M, Vaugeois JM, Nomikos GG, Greengard P (2006) Alterations in 5-HT1B receptor function by 11 in depression-like states. Science 311:77-80.

Svenningsson P, Tzavara ET, Qi H, Carruthers R, Witkin JM, Nomikos GG, Greengard P (2007) Biochemical and behavioral evidence for antidepressant-like effects of 5-HT6 receptor stimulation. J Neurosci 27:4201-4209.

Tonini M, Pace F (2006) Drugs acting on serotonin receptors for the treatment of functional GI disorders. Dig Dis 24:59-69.

Varnäs K, Halldin C, Pike VW, Hall H (2003) Distribution of 5-HT4 receptors in the postmortem human brain-an autoradiographic study using [125I]SB 207710. Eur Neuropsychopharmacol 13:228-234.

Waeber C, Sebben M, Bockaert J, Dumuis A (1996) Regional distribution and ontogeny of 5-HT4 binding sites in rat brain. Behav Brain Res $73: 259-262$.

Warner-Schmidt JL, Duman RS (2006) Hippocampal neurogenesis: opposing effects of stress and antidepressant treatment. Hippocampus 16:239-249. 